\title{
The hole story: an event-related potential study with trypophobic stimuli
}

\author{
Albert Wabnegger $^{1} \cdot$ Daniela Schwab $^{1} \cdot$ Anne Schienle ${ }^{1} \mathbb{C}$
}

Published online: 19 July 2019

(c) The Author(s) 2019

\begin{abstract}
Trypophobia has been defined as the fear and/or aversion of irregular patterns or clusters of small holes. This event-related potential study compared 20 individuals with high trypophobia proneness (TP_high: 16 women, 4 men) and 20 individuals with low TP (16 women, 4 men). All participants viewed images from four different categories: clusters of holes, generally fear-inducing, generally disgust-inducing and neutral pictures. Relative to the TP_low group, the TP_high group showed a larger parietal late positive potential (LPP; $350-900 \mathrm{~ms}$ ) to clusters of holes. The two groups did not differ in their LPP amplitudes to the other image categories. The increased LPP displayed by TP prone individuals reflects facilitated attention to these stimuli of motivational relevance. Clusters of holes primarily elicited disgust in the TP_high group, whereas these stimuli were affectively neutral for the TP_low group.
\end{abstract}

Keywords Trypophobia $\cdot$ Disgust $\cdot$ Event-related potentials $\cdot$ Late positive potential

\section{Introduction}

Specific phobias are the most common mental disorders in the general population with a prevalence of approximately 10\% (American Psychiatric Association 2013). The diagnostic criteria include marked and persistent fear caused by the presence of a specific object or situation. The fear leads to active avoidance of the phobic stimulus, to clinically significant distress and/or impairment in social, occupational, or other areas of functioning. According to the Diagnostic and Statistical Manual of Mental Disorders (DSM-5, American Psychiatric Association 2013) five different subtypes of specific phobias can be distinguished: the animal type, the situational type, the natural environment type, the bloodinjection-injury type and a category labeled 'others'.

Recently, it has been suggested that trypophobia might be such an additional subtype of a specific phobia. Trypophobia

Electronic supplementary material The online version of this article (https://doi.org/10.1007/s11031-019-09784-8) contains supplementary material, which is available to authorized users.

Anne Schienle

anne.schienle@uni-graz.at

1 Clinical Psychology, University of Graz, BioTechMedGraz, Universitätsplatz 2/DG, 8010 Graz, Austria has been defined as the fear and/or aversion of irregular patterns or clusters of small holes (e.g., Cole and Wilkins 2013). Such holes can be found in lotus flowers (seed heads), honeycombs or sponges but are also characteristics of various venomous animals (e.g., certain types of snakes, insects, and spiders). Based on bio-evolutionary models, clusters of holes signal the presence of health-threatening organisms and are therefore able to elicit rejection/withdrawal responses (e.g., Cole and Wilkins 2013). Trypophobia is not yet recognized as a clinically relevant phobia, however, the condition is frequently discussed in the media and surveys point to a high prevalence. In a sample of 195 participants of a trypophobia support group, $31 \%$ reported severe levels of anxiety and somatic distress (e.g., Vlok-Barnard and Stein 2017). Despite the reported frequency and intensity of trypophobic symptoms, the psychological and neurobiological mechanisms of this potential phobic disorder are still poorly understood. Trypophobic stimuli were used in only one event-related potential (ERP) study. Van Strien and van der Peijl (2018) presented students with images depicting clusters of holes, fear-relevant animals (poisonous animals, such as pufferfish, snakes), and neutral animals (birds) in a rapid serial presentation design. They found that holes and poisonous animals evoked more early posterior negativity (EPN) than birds, however less EPN than snakes. The EPN reflects early visual attention and is most pronounced across 
occipital electrodes between 225 and $300 \mathrm{~ms}$ after stimulus onset (Olofsson et al. 2008).

In contrast, numerous studies have focused on the neurobiology of other types of specific phobias (e.g., spider/snake phobia). For example, patients with spider phobia show a typical electrocortical response during the exposure to disorder-relevant stimuli. This response is characterized by enlarged amplitudes of ERPs. The presentation of spider pictures elicited greater parietal P300 amplitudes (ranging from 200 to $520 \mathrm{~ms}$ after stimulus onset), and LPP amplitudes (late positive potential, $550-770 \mathrm{~ms}$ after stimulus onset) in phobics relative to non-phobic controls (e.g., Miltner et al. 2005; Mühlberger et al. 2006; Schienle et al. 2008; Leutgeb et al. 2009; Scharmüller et al. 2011). Late positivity reflects motivated attention (Hajcak et al. 2009; Quiñones-Camacho et al. 2018). Very positive or negative stimuli that induce arousal lead to enlarged late positivity. Therefore, the associated components (P300, LPP) reflect emotional salience (Hajcak et al. 2009). In line with the electrocortical reponse, the patients rated the presented images with spiders as highly fear-inducing and disgusting (Leutgeb et al. 2009; Schienle et al. 2008).

Only a few researchers have addressed the role of fear and disgust in trypophobia. Imaizumi et al. (2016) conducted an online study with 126 adults and found that scores on the Trypophobia Questionnaire (TQ; Le et al. 2015) positively correlated with disgust proneness (DP). DP is a personality trait that reflects a person's tendency to experience disgust across different situations (Schienle et al. 2002). Kupfer and Le (2018) observed that individuals with trypophobia proneness experienced disgust towards disease-relevant as well as disease-irrelevant clusters. The reported aversion was associated with a specific domain of disgust proneness ('pathogen disgust') as revealed by a regression analysis. Ayzenberg et al. (2018) investigated healthy students and showed that images of holes elicited greater pupil constriction than images of threatening animals (e.g., spiders) and neutral images. Constriction of the pupil occurs when the circular muscle, controlled by the parasympathetic nervous system, contracts (Ayzenberg et al. 2018). Parasympathetic activation is typical for disgust (de Jong and Peters 2007). The analysis of the affective ratings however revealed higher fear as well as disgust ratings for animals than holes. Thus, there is still some controversy surrounding the involvement of the two basic emotions fear and disgust in trypophobia.

The current study investigated the role of fear and disgust in trypophobia. The ERP study by van Strien and van der Peijl (2018) only included disorder-relevant and generally fear-inducing images. We presented images of clusters of holes, generally fear-inducing, generally disgust-inducing and neutral pictures (without holes) to participants with high and low scores on a trypophia questionnaire (TQ, Le et al. 2015). We expected that trypophobic participants would show affective modulation of the LPP with the highest amplitude toward clusters of holes (as the disorder-relevant stimulus), followed by fear and disgust pictures (as non-phobic but emotionally relevant stimuli) and finally neutral images. In addition, trait measures of fear and disgust proneness were compared between the two groups in order to further elucidate the role of these basic emotions in trypophobia. Finally, a structured clinical interview was conducted in order to assess whether the diagnostic criteria for a specific phobia of clustes of holes (excessive/persistent fear or disgust, active avoidance, clinically relevant distress and/or functional impairment) would be met by individuals with high TQ scores.

\section{Method}

\section{Participants}

Participants were recruited through advertisements at the university campus. A total of 171 participants completed an online screening with a German translation of the Trypophobia Questionnaire (TQ; Le et al. 2015). A total of 40 participants with the highest and lowest scores on the TQ were invited to the ERP study ( 32 women, 8 men; mean age $=22.87$ years, $\mathrm{SD}=3.34$ ). Twenty participants were characterized by high Trypophobia Proneness (TP_high; TQ scores $\geq 29$ ), whereas the other half was assigned to the TP_low group (TQ scores $\leq 19$ ). Exclusion criteria for participation in the EEG study were neurological disorders and medication. The two groups did not differ in mean age $(p=.79)$, years of education $(p=.96)$ and gender ratio (see Table 1).

\section{Clinical interview and questionnaires}

A board-certified clinical psychologist conducted a structured clinical interview (Mini-DIPS; Margraf 1994) with all participants. The Mini-DIPS screens for anxiety disorders, affective disorders, somatic symptom disorders, eating disorders, substance abuse and psychotic symptoms. In addition, a trypophobia interview (developed by the authors) asked for the diagnostic criteria of a specific phobia according to DSM-5 (marked fear/disgust, active avoidance, clinically significant distress and/or functional impairment elicited by clusters of holes). Each criterion was rated by the participants on 9-point scales ( $1=$ no disgust, fear, avoidance, impairment). Prior to the interview and EEG testing session, the participants completed the following questionnaires via an online screening:

1. The Trypophobia Questionnaire (TQ; Le et al. 2015; German translation) comprises 17 items that assess 
Table 1 Characteristics of the two groups with high and low trypophobia proneness

\begin{tabular}{|c|c|c|}
\hline & $\mathrm{TP} \_$high $\mathrm{M} \pm \mathrm{SD}$ & $\mathrm{TP} \_$low $\mathrm{M} \pm \mathrm{SD}$ \\
\hline Age (years) & $23.02 \pm 3.43$ & $22.7 \pm 3.33$ \\
\hline Gender ratio (female: male) & $16: 4$ & $16: 4$ \\
\hline Years of education & $12.50 \pm 1.50$ & $12.40 \pm 1.47$ \\
\hline Trypophobia proneness (TQ) & $32.95 \pm 3.91$ & $17.25 \pm 0.64$ \\
\hline Trait anxiety (STADI) & $2.44 \pm 0.57$ & $1.76 \pm 0.40$ \\
\hline Trait depression (STADI) & $1.81 \pm 0.51$ & $1.62 \pm 0.42$ \\
\hline Disgust proneness (QADP) & $2.28 \pm 0.45$ & $2.11 \pm 0.56$ \\
\hline $\begin{array}{l}\text { Diagnoses of mental disorders (clinical } \\
\text { interview) }\end{array}$ & $\begin{array}{l}\text { Specific phobia/animal type }(n=6) \text {, natural environment }(n=1) \text {, blood-injection- } \\
\text { injury }(n=1) \text {; obsessive-compulsive disorder (mild symptoms; } n=1) \text {; social } \\
\text { anxiety disorder }(n=1) \text {; major depression (mild symptoms; } n=1)\end{array}$ & None \\
\hline
\end{tabular}

$M$ Means, $S D$ Standard deviations, $T Q$ Trypophobia questionnaire, STADI State-trait-anxiety-depression-inventory, $Q A D P$ Questionnaire for the assessment of disgust proneness

emotional (e.g., repulsion) and somatic responses (e.g., goosebumps) elicited by two images of trypophobic stimuli (lotus seed pods, honey comb). The presence of symptoms is rated on five-point scales ranging from 1 ("not at all") to 5 ("extremely"). The sum score reflects trypophobia proneness. The Cronbach's $\alpha$ of the scale was .88 in the present sample.

2. The Questionnaire for the Assessment of Disgust Proneness (QADP; Schienle et al. 2002) assesses the general tendency of a person to experience disgust across different situations (e.g., 'You are just about to drink a glass of milk as you notice that it is spoiled'). The items are rated on 5-point scales $(0=$ not disgusting; $4=$ very disgusting). The Cronbach's $\alpha$ of the scale was .87 .

3. The trait section of the State-Trait-Anxiety-DepressionInventory (STADI; Laux et al. 2013) consists of four trait subscales (Arousal, Worrying, Dysthymia, Euthymia), each with five items. Trait Anxiety is comprised of Arousal (e.g., I'm getting nervous quickly) and Worrying, (e.g., I worry that something might happen); Trait Depression is determined based on the two subscales Dysthymia (e.g., I am sad) and Euthymia (e.g., I enyoy life). The items are rated on 4-point scales $(1=$ not all all, $4=$ very much; scores for Euthymia are inverted). Higher sum scores suggest higher levels of trait anxiety/ trait depression. The Cronbach's alphas of the two trait scales were $.88 / .89$ in the present sample.

\section{Stimuli}

The participants viewed a total of 120 pictures from four categories 'Holes', 'Disgust', 'Fear', and 'Neutral' (with 30 pictures each). Pictures depicting clusters of holes (e.g., lotus seed pods, sponges, corals, stone formations) were taken from the Internet and are comparable to the stimuli used by (Le et al. 2015). Generally fear-eliciting pictures (e.g., lions, sharks, weapons, aggression by humans), pictures with neutral items (e.g., household articles) and generally disgust-eliciting pictures (e.g., poor hygiene, spoiled food) were either taken from the International Affective Picture System (Lang et al. 2008) $)^{1}$ or a validated picture set (disgust, fear, neutral) by Schienle et al. 2002).

\section{Procedure and design}

All participants completed the TQ, QADP, and STADI in an online survey. Then, the participants with the highest and lowest TQ scores were invited to a clinical interview. During the subsequent EEG session, the pictures ('Holes', 'Disgust', 'Fear', and 'Neutral') were displayed on a computer screen (diagonal: $22 \mathrm{inch}$; eye distance to the screen $=110 \mathrm{~cm}$ ). The participants were instructed to passively view the images, which were presented in randomized order for $1500 \mathrm{~ms}$ each. Each image was preceded by a fixation cross (500$1000 \mathrm{~ms}$ ). Five pictures per category were evaluated by the participants according to experienced disgust and fear on nine-point Likert scales $(1=$ not at all, $9=$ very much $)$ during the experiment.

The study was conducted in accordance with the Declaration of Helsinki and approved by the ethics committee of the University. All participants gave written informed consent.

\section{Electrophysiological recordings and data analyses}

Data were recorded with a Brain Amp 32 System (32 channel amplifier, Brain Vision Recorder Version 1.2; Brain Products, Gilching). Ag/AgCl electrodes were placed according to 10-20 system using an Easy Cap multitrode

\footnotetext{
${ }^{1}$ IAPS numbers (Fear: 0012, 1726, 1932, 6350, 0009, 0014, 0015, 0016, 1300, 1302, 1303, 1321, 1930, 2690, 3500, 3530, 6211, 6212, 6230, 6250, 6312, 6370, 6510, 6530, 6540, 6560; Disgust: 9300; Neutral: 5395, 7000, 7009, 7034, 7080, 7096, 7100, 7175, 7211, 7491, 7140).
} 
system (EASYCAP, Herrsching) on 31 positions (FP1, FP2, F7, F3, Fz, F4, F8, FC5, FC1, FC2, FC6, T7, C3, Cz, C4, T8, CP5, CP1, CP2, CP6, P7, P3, Pz, P4, P8, O1, O2, FT9, FT10, TP9, TP10). One electrode was placed supraorbital of the right eye in order to record vertical eye movement. Electrodes at FT9 and FT10 recorded horizontal eye movements. The reference electrode was placed on position $\mathrm{FCz}$, the ground electrode on position FPz. The scalp was gently abraded with chloride-free electrolyte gel to keep electrode impedances below $5 \mathrm{k} \Omega$. The sampling rate was $2500 \mathrm{~Hz}$ with a passband of $0.016-1000 \mathrm{~Hz}$. For raw data analysis, the Brain Vision Analyzer (Version 2.0.4) was used. The sampling rate was set to $250 \mathrm{~Hz}$. The data were re-referenced to averaged mastoid electrodes (TP9, TP10). Artifacts due to eye movements were corrected via the implemented ICA ocular correction software. Further artifact episodes (e.g., due to swallowing) were excluded after visual inspection (percentage of artifact-free trials: $96.2 \%$; percentage of artifact-free trials did not differ between groups and conditions: all $p>.22$ ). The data were segmented in $1700 \mathrm{~ms}$ intervals (200 ms pre-stimulus, $1500 \mathrm{~ms}$ post-stimulus) and corrected to the $200 \mathrm{~ms}$ pre-stimulus baseline (i.e. subtraction of the averaged baseline from all points in the post-stimulus segment). A $30 \mathrm{~Hz}$ low-pass filter was applied.

Time windows and positions for early and late ERPs were selected based on previous research on specific phobia (e.g., Leutgeb et al. 2009). The selection was validated based on visual inspection of the grand averages and cortical maps. The following ERPs were analyzed: EPN/P100 (100-150 ms and 250-350 ms at O1, O2); N100/P200/N200 (100-150 ms, 150-200 ms and 250-350 ms at F3, Fz, F4), early and late LPPs (350-900 ms (see figure S1) and 900-1500 ms at P3, $\mathrm{Pz}, \mathrm{P} 4)$. The averaged activity in each cluster was used for statistical analyses.

The analyses showed no significant group or interaction effects for early components (P100, N100, P200, N200, EPN) and the late LPP. Therefore, these results are not reported.

\section{Statistical analysis}

Group differences regarding questionnaire scores were analyzed by means of independent t-tests. Analyses of variance (ANOVAs) with the between-subject factor GROUP (TP high, TP_low) and the within-subject factor CATEGORY (Holes, Disgust, Fear, Neutral) were computed for affective picture ratings and ERP data. If sphericity violations (Mauchly test) occurred, Greenhouse-Geisser-corrected degrees of freedom are reported. Significant main effects were followed by post hoc t-tests. Effect sizes are expressed by partial eta squared $\left(\eta p^{2}\right)$ or Cohen's d. All statistical analyses were conducted with SPSS 25.

\section{Results}

\section{Questionnaires and clinical interview}

The TP_high group reported higher trypophobia proneness $(\mathrm{t}(22.01)=-17.71, \mathrm{p}<.001 ; \mathrm{d}=-5.60)$, and higher levels of trait anxiety $(\mathrm{t}(38)=-4.35, \mathrm{p}<.001 ; \mathrm{d}=-1.38)$ than the TP_low group. The groups did not differ in trait depression $(\mathrm{t}(38)=-1.28, \mathrm{p}=.21 ; \mathrm{d}=-0.41)$, and disgust proneness $(\mathrm{t}(38)=-1.03, \mathrm{p}=.31 ; \mathrm{d}=-0.32$; for descriptives see Table 1). Of the TP_high group, one patient had a diagnosis of obsessive-compulsive disorder and another reported mild depressive symptoms. The exclusion of these patients from the sample did not change the results (self-report, ERP findings). Therefore, we report findings for all subjects $(n=40)$.

In the clinical interview, the participants of the TP_high group named disorder-relevant clusters, which included lotus seeds, photomontage of clusters of holes on skin, honeycombs, stone formations (pumice), sponges, cheese, froth, and noodles. The clusters provoked more disgust $(\mathrm{M}=5.30, \mathrm{SD}=1.92)$ than fear $(\mathrm{M}=2.18, \mathrm{SD}=1.82$; $(t(19)=5.10, p<.001 ; d=1.14)$. The reported degree of functional impairment $(\mathrm{M}=1.93, \mathrm{SD}=1.89)$ and the degree of active avoidance $(\mathrm{M}=1.68, \mathrm{SD}=1.36)$ were low.

\section{Affective picture ratings}

\section{Disgust ratings}

The ANOVA revealed significant main effects of GROUP $\left(\mathrm{F}(1,38)=11.85, \mathrm{p}=.001, \eta \mathrm{p}^{2}=.24\right)$ and CATEGORY $\left(\mathrm{F}(3114)=159.20, \mathrm{p}<.001, \eta \mathrm{p}^{2}=.81\right)$. The TP_high group experienced more disgust than the TP_low group. Disgust images received the highest disgust ratings compared to fear pictures and pictures of holes which in turn were rated as more disgusting than neutral pictures (see Table 2 and supplementary Table S1).

Additionally, the interaction effect GROUP x CATEGORY was significant $\left(\mathrm{F}(3114)=15.06, \mathrm{p}<.001, \eta \mathrm{p}^{2}=.28\right)$. The TP_high group rated clusters of holes and generally disgusting contents as more disgusting than the TP_low group. In addition, the TP_high group gave the highest ratings for disgust pictures followed by pictures of holes which in turn received higher disgust ratings compared to fear pictures. Neutral pictures received the lowest disgust ratings. The TP low group gave the highest ratings for disgust pictures followed by fear pictures which in turn received higher disgust ratings compared to pictures of holes and neutral pictures (see Table 2 and supplementary Table S1). 

(1-9), and LPP amplitudes for the four picture categories (means and standard deviations)
Table 2 Disgust, fear ratings

\begin{tabular}{|c|c|c|c|c|c|}
\hline & Holes & Disgust & Fear & Neutral & All \\
\hline \multicolumn{6}{|l|}{ ТP } \\
\hline \multicolumn{6}{|c|}{ Disgust ratings $(\mathrm{M} \pm \mathrm{SD})$} \\
\hline High & $3.85 \pm 1.73$ & $6.61 \pm 1.43$ & $2.08 \pm 1.11$ & $1.33 \pm 0.78$ & $3.47 \pm 1.27$ \\
\hline Low & $1.42 \pm 0.76$ & $4.93 \pm 1.75$ & $2.06 \pm 1.41$ & $1.28 \pm 0.80$ & $2.42 \pm 1.18$ \\
\hline Total & $2.64 \pm 1.81$ & $5.77 \pm 1.79$ & $2.07 \pm 1.26$ & $1.31 \pm 0.78$ & \\
\hline \multicolumn{6}{|c|}{ Fear ratings $(\mathrm{M} \pm \mathrm{SD})$} \\
\hline High & $2.69 \pm 1.40$ & $3.00 \pm 1.72$ & $4.30 \pm 2.20$ & $1.34 \pm 0.52$ & $2.83 \pm 1.46$ \\
\hline Low & $1.28 \pm 0.67$ & $1.65 \pm 0.96$ & $3.38 \pm 1.91$ & $1.18 \pm 0.43$ & $1.87 \pm 1.00$ \\
\hline Total & $1.99 \pm 1.30$ & $2.33 \pm 1.91$ & $3.84 \pm 2.09$ & $1.26 \pm 0.48$ & \\
\hline \multicolumn{6}{|c|}{ LPP amplitude $(\mathrm{M} \pm \mathrm{SD})$} \\
\hline High & $3.35 \pm 2.88$ & $3.72 \pm 3.03$ & $4.40 \pm 2.73$ & $1.27 \pm 2.42$ & $3.19 \pm 2.54$ \\
\hline Low & $1.03 \pm 2.70$ & $2.23 \pm 2.61$ & $3.19 \pm 2.61$ & $0.55 \pm 2.32$ & $1.75 \pm 2.30$ \\
\hline Total & $2.19 \pm 3.00$ & $2.98 \pm 2.89$ & $3.80 \pm 2.71$ & $0.91 \pm 2.37$ & \\
\hline
\end{tabular}

TP Trypophobia proneness

\section{Fear ratings}

The ANOVA revealed significant main effects of GROUP $\left(\mathrm{F}(1,38)=8.64, \mathrm{p}=.006, \eta \mathrm{p}^{2}=.19\right)$ and CATEGORY $\left(\mathrm{F}(1.92,73.03)=42.05, \mathrm{p}<.001, \eta \mathrm{p}^{2}=.53\right)$. The TP_high group experienced more fear than the TP_low group. The fear pictures received higher fear ratings compared to disgust pictures which in turn were rated as more fear eliciting than pictures of holes. Neutral pictures received the lowest fear ratings (see Table 2 and supplementary Table $\mathrm{S} 1$ ). The interaction effect was not significant $(\mathrm{F}(1.92,73.03)=2.96$, $\mathrm{p}=.06, \eta \mathrm{p}^{2}=.07$, see Table 2).

\section{Early LPP (350—900 ms)}

The main effect CATEGORY $(\mathrm{F}(3114)=36.29, \mathrm{p}<.001$, $\left.\eta \mathrm{p}^{2}=.49\right)$ and the interaction effect GROUP $\mathrm{x}$ CATEGORY were significant $\left(\mathrm{F}(3114)=2.70, \mathrm{p}=.049, \eta \mathrm{p}^{2}=.07\right)$. The main effect GROUP was not significant $(\mathrm{F}(1,38)=3.50$, $\left.\mathrm{p}=.07, \eta \mathrm{p}^{2}=.08\right)$. The LPP amplitude to fear pictures was larger compared to pictures of holes, which in turn was larger compared to disgust pictures. The LPP amplitude to neutral pictures was the smallest (see Table 2 and supplementary Table S1).

The LPP amplitude for clusters of holes in the TP_high group was significantly larger than in the TP_low group (see Fig. 1). Within the TP high group, the LPP to fear pictures was larger compared to pictures of holes. LPP amplitudes for holes, fear and disgust pictures were increased relative to neutral pictures. Within the TP_low group, the LPP to fear pictures was larger compared to disgust pictures. The presentation of disgust pictures provoked a higher LPP amplitude than neutral pictures and holes. There was no difference in the LPP amplitude between pictures depicting holes and neutral content.

\section{Discussion}

This ERP study focused on the role of fear and disgust in trypophobia. We compared participants with high versus low cal responses to four types of affective pictures (clusters of holes, fear, disgust, neutral).

The ERP analysis indicated that the TP_high group (relative to TP_low) was characterized by increased parietal late positive potentials (LPP: $350-900 \mathrm{~ms}$ ) in response to images of holes. The LPP is an event-related potential that is enhanced when viewing (un)pleasant pictures compared to neutral pictures (e.g., Hajcak et al. 2009; Schupp et al. 2006). The affective modulation of the LPP (especially of the early component) is believed to reflect increased motivated attention (Hajcak et al. 2009; Olofsson et al. 2008) which facilitates perceptual processing and subsequent adaptive responses to these emotional stimuli (e.g., approach/ withdrawal behaviors). The ERP data of the present study point to greater emotional salience of clusters of holes for trypophobia-prone individuals.

The affective picture ratings and the clinical interview gave further insights into the emotional impact of the clusters of holes. TP prone participants described lotus seeds, honeycombs and stone formations with holes as relevant triggers of their symptoms. These stimuli predominantly elicited disgust (and not fear). In this context, it has to be noted that many participants stated that the photomontages of holes projected onto skin were the most important elicitors of their aversion. Such photos are shared on social media, either from people with questions about the condition or from those who look to cause disturbing reactions in others. The artificial combination of skin and holes creates the impression of a pathological skin condition. Yamada and Sasaki (2017) proposed the 'involuntary protection against trypophobia proneness in their affective and electrocorti- 

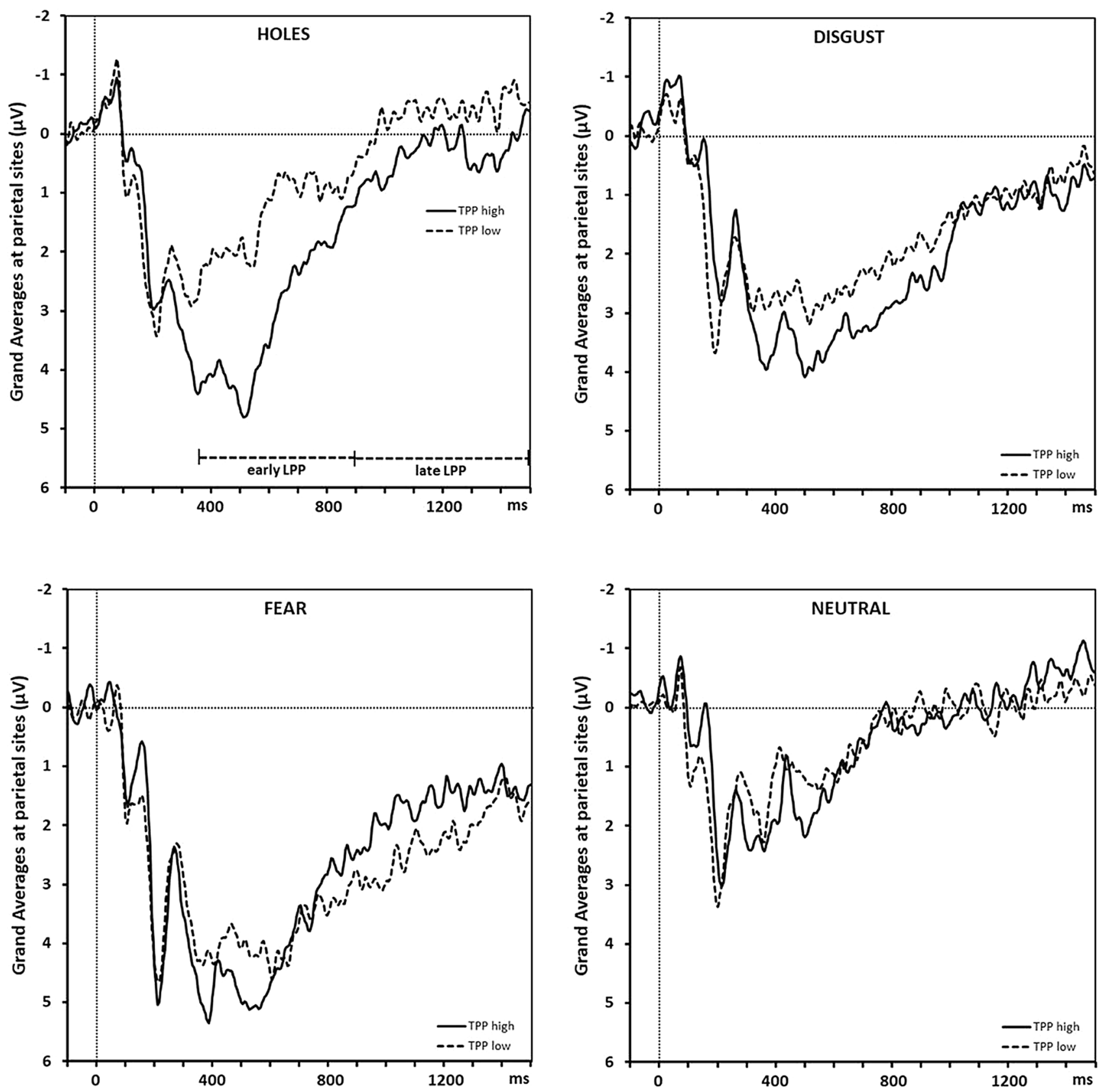

Fig. 1 Grand averages at parietal sites comparing the TP_high and TP_low group for each picture category

dermatosis' (IPAD) hypothesis of trypophobia. The authors noted that contagious skin diseases have similar visual patterns like trypophobic stimuli. Yamada and Sasaki (2017) found that images with clusters of holes elicited greater discomfort in individuals with a history of skin-related medical problems than in those without such problems. In this sense, trypophobic tendencies might index an elevated intuitive sense of what can cause (skin) infection. In line with this model, Kupfer and Le (2018) conceptualized trypophobia as an overgeneralized disease avoidance response. This implies elevated disgust proneness for a specific stimulus category ('disease') but no generally elevated DP, which was also found in the current investigation.

The trypophobia-prone participants reported elevated trait anxiety and rated pictures of all categories as more fear-eliciting. Moreover, some participants with high TP had a diagnosis of an anxiety disorder (most often spider phobia). In contrast to the self-report data, the EEG data did not reflect this elevated anxiety proneness of the TP_high group. The LPP amplitudes to the fear pictures did not differ between the two groups. Within this context, it has to be noted that the selected fear images showed scenes of human 
aggression and attacking predators but no phobia-relevant animals (e.g., spiders).

Contrary to our hypothesis, the TP_high group did not display the highest LPP amplitude to clusters of holes (but to fear pictures). This response pattern is surprising because phobic individuals typically show most pronounced late positivity to the disorder-relevant stimulus (e.g., Miltner et al. 2005; Mühlberger et al. 2006; Leutgeb et al. 2009; Scharmüller et al. 2011; Schienle et al. 2008). For example, in a study by Leutgeb et al. (2009), spider phobics were characterized by LPP amplitudes to pictures of spiders that were almost twice as high compared to generally fear-relevant and disgust-relevant pictures. Our findings do not show an electrocortical pattern that is typical for patients with clinically relevant phobic symptoms. In line with this observation, the clinical interview showed that none of the participants with a high TQ score met the diagnostic criteria of specific phobia of clusters of holes, because levels of avoidance and functional impairment were low. Therefore, these participants received the label 'high trypophobia proneness'. This label reflects the pronounced distress during the confrontation with clusters of holes but also indicates that no phobia was present.

In conclusion, this ERP study revealed that clusters of holes are affectively neutral for some individuals, whereas others find these stimuli very disgusting. The disgust-inducing properties of clusters of holes were associated with elevated parietal late positivity reflecting increased motivated attention.

Acknowledgements Open access funding provided by University of Graz.

\section{Compliance with ethical standards}

Conflict of interest The authors have no competing interests to declare.

Ethical approval All procedures performed in this study were in accordance with the ethical standards of the institutional research committee (approved by the ethics committee of the University) and with the 1964 Helsinki declaration and its later amendments.

Informed consent All participants gave their written informed consent.

Open Access This article is distributed under the terms of the Creative Commons Attribution 4.0 International License (http://creativeco mmons.org/licenses/by/4.0/), which permits unrestricted use, distribution, and reproduction in any medium, provided you give appropriate credit to the original author(s) and the source, provide a link to the Creative Commons license, and indicate if changes were made.

\section{References}

American Psychiatric Association. (2013). Diagnostic and statistical manual of mental disorders: DSM-5 (5th ed). Washington DC: American Psychiatric Association.

Ayzenberg, V., Hickey, M. R., \& Lourenco, S. F. (2018). Pupillometry reveals the physiological underpinnings of the aversion to holes. PeerJ, 6, e4185. https://doi.org/10.7717/peerj.4185.

Cole, G. G., \& Wilkins, A. J. (2013). Fear of holes. Psychological Science, 24(10), 1980-1985. https://doi.org/10.1177/0956797613 484937.

De Jong, P. J., \& Peters, M. L. (2007). Blood-injection-injury fears: Harm- versus disgust-relevant selective outcome associations. Journal of Behavior Therapy and Experimental Psychiatry, 38(3), 263-274.

Hajcak, G., Dunning, J. P., \& Foti, D. (2009). Motivated and controlled attention to emotion: Time-course of the late positive potential. Clinical Neurophysiology, 120(3), 505-510. https:// doi.org/10.1016/j.clinph.2008.11.028.

Imaizumi, S., Furuno, M., Hibino, H., \& Koyama, S. (2016). Trypophobia is predicted by disgust sensitivity, empathic traits, and visual discomfort. SpringerPlus, 5(1), 1449. https://doi. org/10.1186/s40064-016-3149-6.

Kupfer, T. R., \& Le, A. T. D. (2018). Disgusting clusters: Trypophobia as an overgeneralised disease avoidance response. Cognition and Emotion, 32(4), 729-741. https://doi.org/10.1080/02699 931.2017.1345721.

Lang PJ, Bradley MM, Cuthbert BN (2008) International affective picture system (IAPS): Affective ratings of pictures and instruction manual. Technical report A-8

Laux, L., Hock, M., Bergner-Köther, R., Hodapp, V., \& Renner, K.-H. (2013). STADI: Das State-Trait-Angst-Depressions-Inventar. Göttingen: Hogrefe Verlag.

Le, A. T. D., Cole, G. G., \& Wilkins, A. J. (2015). Assessment of trypophobia and an analysis of its visual precipitation. Quarterly Journal of Experimental Psychology, 68(11), 2304-2322.

Leutgeb, V., Schäfer, A., \& Schienle, A. (2009). An event-related potential study on exposure therapy for patients suffering from spider phobia. Biological Psychology, 82(3), 293-300. https:// doi.org/10.1016/j.biopsycho.2009.09.003.

Margraf, J. (1994). Mini-DIPS: Diagnostisches Kurz-Interview bei psychischen Störungen [Diagnostic short-interview for psychic problems]. Berlin: Springer.

Miltner, W. H. R., Trippe, R. H., Krieschel, S., Gutberlet, I., Hecht, H., \& Weiss, T. (2005). Event-related brain potentials and affective responses to threat in spider/snake-phobic and non-phobic subjects. International Journal of Psychophysiology, 57(1), 43-52. https://doi.org/10.1016/j.ijpsycho.2005.01.012.

Mühlberger, A., Wiedemann, G., Herrmann, M. J., \& Pauli, P. (2006). Phylo- and ontogenetic fears and the expectation of danger: Differences between spider- and flight-phobic subjects in cognitive and physiological responses to disorder-specific stimuli. Journal of Abnormal Psychology, 115(3), 580-589. https://doi.org/10.1037/0021-843X.115.3.580.

Olofsson, J. K., Nordin, S., Sequeira, H., \& Polich, J. (2008). Affective picture processing: an integrative review of ERP findings. Biological Psychology, 77(3), 247-265.

Quiñones-Camacho, L. E., Wu, R., \& Davis, E. L. (2018). Motivated attention to fear-related stimuli: Evidence for the enhanced processing of fear in the late positive potential. Motivation and Emotion, 42(2), 299-308. https://doi.org/10.1007/s1103 1-018-9670-x.

Scharmüller, W., Leutgeb, V., Schäfer, A., Köchel, A., \& Schienle, A. (2011). Source localization of late electrocortical positivity during symptom provocation in spider phobia: An sLORETA 
study. Brain Research, 1397, 10-18. https://doi.org/10.1016/j. brainres.2011.04.018.

Schienle, A., Schäfer, A., \& Naumann, E. (2008). Event-related brain potentials of spider phobics to disorder- relevant, generally disgust- and fear-inducing pictures. Journal of Psychophysiology, 22(1), 5-13. https://doi.org/10.1027/0269-8803.22.1.5.

Schienle, A., Stark, R., Walter, B., Blecker, C., Ott, U., Kirsch, P., et al. (2002a). The insula is not specifically involved in disgust processing: An fMRI study. NeuroReport, 13(16), 2023-2026. https://doi. org/10.1097/00001756-200211150-00006.

Schienle, A., Walter, Bertram, Stark, Rudolf, \& Vaitl, D. (2002b). Ein Fragebogen zur Erfassung der Ekelempfindlichkeit (FEE). Zeitschrift für Klinische Psychologie und Psychotherapie, 31(2), 110-120.

Schupp, H. T., Flaisch, T., Stockburger, J., \& Junghöfer, M. (2006). Emotion and attention: Event-related brain potential studies. In S. Anders, G. Ende, \& M. Junghofer (Eds.), Progress in Brain
Research: Understanding Emotions (pp. 31-51). Amsterdam: Elsevier.

Van Strien, J., \& van der Peijl, M. K. (2018). Enhanced early visual processing in response to snake and trypophobic stimuli. $B M C$ Psychology, 6(1), 21. https://doi.org/10.1186/s40359-018-0235-2.

Vlok-Barnard, M., \& Stein, D. J. (2017). Trypophobia: An investigation of clinical features. Revista Brasileira De Psiquiatria, 39(4), 337-341.

Yamada, Y., \& Sasaki, K. (2017). Involuntary protection against dermatosis: A preliminary observation on trypophobia. BMC Research Notes, 10(1), 658. https://doi.org/10.1186/s13104-017-2953-6.

Publisher's Note Springer Nature remains neutral with regard to jurisdictional claims in published maps and institutional affiliations. 